\title{
Statistical Characteristics of English Entrance Exams of National Universities in Japan
}

\author{
Kiyomi Watanabe \\ Business Administration Dept, Fukuyama Heisei University, \\ Miyuki-cho, Fukuyama-city, Japan

\section{Masayasu Fukui} \\ Business Administration Dept, Fukuyama Heisei University, \\ Miyuki-cho, Fukuyama-city, Japan \\ Mitsuhiro Hosokawa \\ University Education Center, Fukuyama Heisei University, \\ Miyuki-cho, Fukuyama-city, Japan
}

\begin{abstract}
In spite of the decreasing population of teenagers in Japan, the university entrance exams continue to be one of the toughest events where their intelligence is tested, which include their competency level of English. However, very few researches have conducted on English exams. This study is a part of the continuous study the researchers have started in 2017, which studied the English entrance exams of eight national universities. They were selected from the list according to the T-score; two schools from 4 levels. The current study added the results of 2018 exams of the same universities. Upon digitalizing their English exams, the researchers first measured their readability levels using Ozasa-Fukui Year Level. They also performed Correspondence Analysis to study the interrelations among the nine exams. All eight exams are measured to be $7^{\text {th }}$ year or higher by Ozasa-Fukui Year Level, which is created to measure English level according to the 6 years in the secondary school system in Japan. The correspondence analysis produced 7 dimensions. However, due to the unexpectedly high value of one exam, the other seven exams' interrelations became obscured; we have decided to perform another analysis without that particular exam. Its results showed that the seven exams could be categorized according to "business and culture" and "family." The researchers further employed Cluster Analysis to study all seven dimensions produced by Correspondence Analysis, which showed that Kyoto18 and Hokkaido18 have strong ties with each other. We believe this study has given us a definite starting point for the research on the university entrance exams in Japan.
\end{abstract}

Keywords: readability, university entrance exam, correspondence analysis. 


\section{Introduction}

\section{National University Entrance Exams in Japan}

Although it is often pointed out that university entrance exams are not as competitive as they once were due to the rapid decrease of the teenage population, they are considered as many as the toughest test of a lifetime. The level of entrance exams of the top universities exceeds the guidelines provided by the Course of Study of the Ministry of Education in Japan, and the guidelines, which authorized textbooks for secondary school, must follow. Various publishers publish lists of top universities every year, which is based on a T-score. Although the order differs a little among publishers, the basic order remains the same: Tokyo University comes first, followed by Kyoto University and others.

The system of university entrance exams has two parts. The first part is the National Center Test for University Admission (the Center Test); the first round of standardized entrance exam for national universities, and the second is the entrance exams each national university gives. Although there is no "pass" or "fail" for the National Center Test, some top ranking universities often set score limits as an entry requirement for applying to those universities. As for the second round of tests, every year, each university makes a special effort to create its exam difficult enough to allow them to select the best students with high intelligence.

\section{Significance and Aim}

Although many careful studies have been done and published on the tendency and particular features of each exam for the use of the candidates, only a few academic studies have been done to examine the exams from a textual analysis point of view. One of the possible reasons for this is that the data is too big to be digitalized; there are 779 universities in Japan (86 national universities, 89 local public universities, and 604 private universities) (the data provided by the Ministry of Education), not to mention that they make their own exams every year.

Watanabe \& Fukui (2018) selected eight national universities from the list according to the T-score and studied their English entrance exams. The result of English readability measurement using Ozasa-Fukui Year Level, a digital tool to measure English sentences according to the 6 years of the secondary school system in Japan, showed that all eight exams are higher than the sixth year level (the third year of high school).

In regards to previous research on university entrance exams, we found a few of them. Hasegawa, Nakajyo, and Nishigaki (2006) examined the vocabulary used in exams. Urabe (2010) studied compositions test in university entrance exams. Moreover, Ide (2012) studied entrance exams of the east-Asian countries in comparison with the Center Test of Japan.

A few studies have done on English textbooks and they clearly indicate that statistical analyses are good tools for comparative studies because they show the relationships quantitatively among textbooks and can produce specific features of each textbook (Ozasa \& Abe, 2015; Ozasa, Watanabe, Uenishi \& Sakamoto, 2016; Ozasa, Watanabe, Uenishi \& Sakamoto, 2017; Uenishi, Watanabe \& Ozasa, 2017; Sakamoto, Watanabe \& Ozasa, 2017). These authors studied the readability level of English textbooks in Asia in comparison to Japanese English textbooks. Both Uenishi, Watanabe \& Ozasa (2017) and Sakamoto, Sakamoto, Watanabe, \& Ozasa (2017) employ correspondence analysis to study the historical Japanese English textbooks, of which results parallel the readability results. Watanabe \& Fukui (2018) studied the first-year English textbooks of China, Thailand, Japan, and the Philippines and found that their readability levels correspond with the correspondence analysis. All these previous studies have shown that the statistical analyses employed in the analyses successfully produced 
some clear features and characteristics of the target texts. Therefore, the aim of the current study on the university English entrance exams is to investigate the English sentences used in these exams to find specific features and characteristics of the exams, and to study if these features and characteristics would correspond with the order of the T-score of the national universities.

The current study is a follow-up of Watanabe \& Fukui (2018); in addition to 2017 exams data, we include 2018 data of the same universities. We set the following research questions to describe the aim of the study.

RQ1: What are 2018 exams' readability levels?

RQ2: Are there any common characteristics between 2017 and 2018 exams of the same school? RQ3: Are there any characteristics unique to 2017 or to 2018 ?

Table 1 shows the list of universities we have selected for the analysis and their T-scores.

Table 1: T-Score

\begin{tabular}{|l|l|}
\hline $\begin{array}{l}\text { T- } \\
\text { score }\end{array}$ & University \\
\hline \multirow{2}{*}{$75 \sim$} & Tokyo University \\
\cline { 2 - 2 } & Kyoto University \\
\hline \multirow{2}{*}{$70 \sim 74$} & Osaka University \\
\cline { 2 - 2 } & Kyushu University \\
\hline \multirow{2}{*}{$65 \sim 69$} & Tohoku University \\
\cline { 2 - 2 } & Hokkaido University \\
\hline \multirow{2}{*}{$60 \sim 64$} & Osaka City University \\
\cline { 2 - 2 } & Hiroshima University \\
\hline
\end{tabular}

* T-score list provided by Benesse (2018) Corporation

We used Orasa-Fukui Year Level Ver. 3.4.2nhnc1-6 $\left(\mathrm{R}^{\wedge} 2=0.88\right)$ (OFYL) as the main measurement tool (Ozasa \& Fukui, 2017). These readability tool measures English sentences according to the Japanese school grade level from grade 7-12. We also used Flesch Reading Ease and Flesch-Kincaid Grade Level as supplementary data because they are designed to measure according to the grade level of U.S. schools. We also compared their vocabulary with JACET8000, a graded vocabulary list for colleges in Japan.

As for extracting unique characteristics of each exam, we used Correspondence Analysis (CA). CA is a data reduction procedure similar to factor analysis. It describes the relationships among nominal variables (in this study, 16 exams) while simultaneously describing the relationships between the nominal dimensions for each variable. Its results are often displayed in a two-dimensional graphical map since the first and second dimensions have the highest contribution rate among all of the dimensions. Ishikawa, Maeda, Yamazaki (2010) describes CS as one of the handiest statistical analyses for categorizing textual data (p. 245). 


\section{Readability Analysis}

\section{Results}

We used Orasa-Fukui Year Level Ver. 3.4 .2 nhnc1-6 $\left(\mathrm{R}^{\wedge} 2=0.88\right)$ as the main measurement tool (Ozasa \& Fukui, 2017). This readability tool measures English sentences according to the grade level of Japanese schools from grade 7-12. We also used Flesch Reading Ease and Flesch-Kincaid Grade Level as supplementary tools; as much as both of them are useful measurement tools, they are designed to measure according to the grade level of American grade schools (Flesh Reading Ease measures sentences on a 100 point-scale; the higher the score the easier).

Table 2: Readability score

\begin{tabular}{|c|c|c|c|c|c|c|c|c|}
\hline & & & $\begin{array}{l}\text { Ozas } \\
\text { Level }\end{array}$ & kui & $\begin{array}{l}\text { Flesch } \\
\text { Ease }\end{array}$ & $\operatorname{Re}$ & $\begin{array}{l}\text { Flesch } \\
\text { Grade }\end{array}$ & $\begin{array}{l}\text { incaid } \\
\text { evel }\end{array}$ \\
\hline & T-score & Universities & 2017 & 2018 & 2017 & 2018 & 2017 & 2018 \\
\hline 1 & & Tokyo University & 7.79 & 8.01 & 37.41 & 44.90 & 10.71 & 10.42 \\
\hline 2 & & Kyoto University & 8.27 & 7.43 & 18.73 & 58.65 & 10.64 & 9.63 \\
\hline 3 & & Osaka University & 7.41 & 7.73 & 43.4 & 47.87 & 9.72 & 9.91 \\
\hline 4 & & Kyushu Uvniersity & 7.56 & 6.76 & 37.9 & 59.70 & 10.02 & 8.27 \\
\hline 5 & & Tohoku Unviersity & 7.89 & 8.17 & 47.53 & 39.88 & 9.9 & 11.03 \\
\hline 6 & & Hokkaido University & 7.64 & 6.99 & 48.93 & 54.58 & 9.34 & 9.03 \\
\hline 7 & & Osaka City University & 7.34 & 7.33 & 58.21 & 65.44 & 8.17 & 8.04 \\
\hline 8 & & Hiroshima University & 8.61 & 6.98 & 28.73 & 52.19 & 11.06 & 9.09 \\
\hline
\end{tabular}

We also compared the exams with JACET 8000, a standardized vocabulary list with 8 levels for colleges in Japan. Figure 1 (2017) and Figure 2 (2018) shows their levels.

Figure 1: 2017 vocabulary levels according to JACET 8000

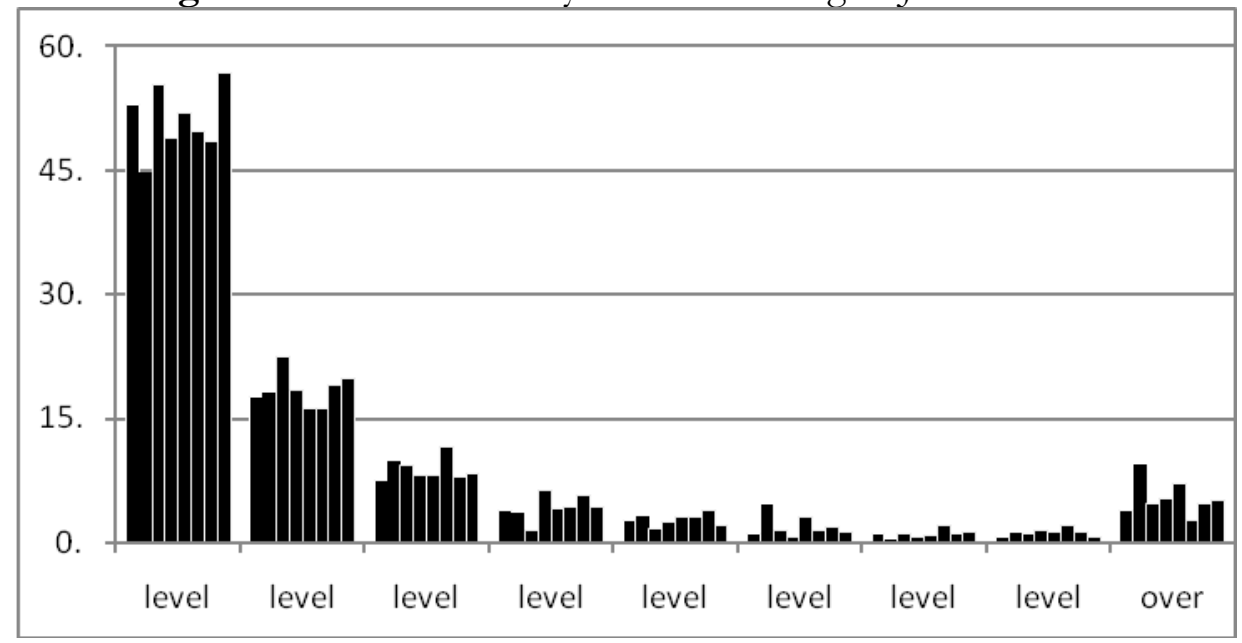

(From left to right: Tokyo17, Kyoto17, Kyusyu17, Osaka17, Tohoku17, Hokkaido17, Hiroshima17, Osaka-City17) 
Figure 2: 2018 vocabulary levels according to JACET8000

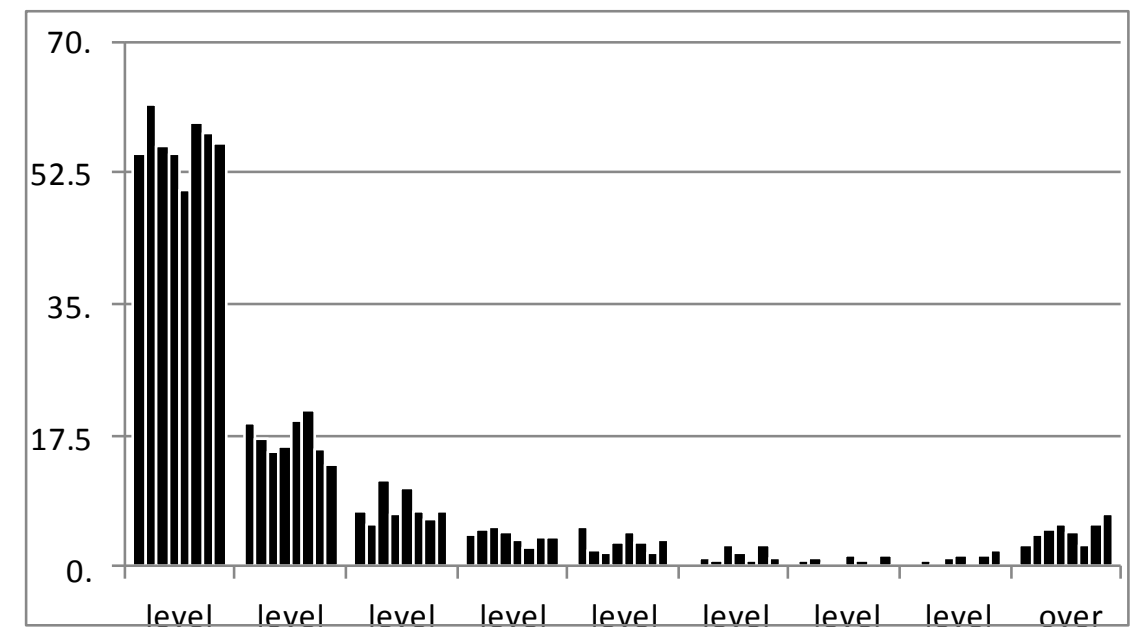

(From left to right: Tokyo18, Kyoto18, Kyusyu18, Osaka18, Tohoku18, Hokkaido18, Hiroshima18, Osaka-City18)

\section{Discussion}

OFYL measures English sentences according to the 6 years of the secondary schools in Japan, of which English curriculum follows the Course of Study prepared by the Ministry of Education: each year is divided into 10 levels, for instance, 1.0-1.9 refers to the $1^{\text {st }}$ year of junior high school. In Table 2, all of the 8 universities' OFYL levels with the exception of 3 schools in 2018 are higher than 7.0 meaning all are much higher than the $3^{\text {rd }}$ year of high school. While the OFYL levels are not exactly in parallel with FRE or FKGL, which is understandable because for one thing, OFYL measures not according to the difficulty of sentences but according to the Course of Study, and for the second reason, since OFYL measures the 6 years of secondary schools (from 1.0 to 6.9 ) so any levels above 6.9 are not reliable. Consequently, the eight universities' OFYL levels are not statistically reliable. We have pointed out in Watanabe \& Fukui (2018) that the English exams of the eight national universities contain sentences that are well above the third year level of high school so the current study shows very similar results.

Table 3: Readability score with ranking

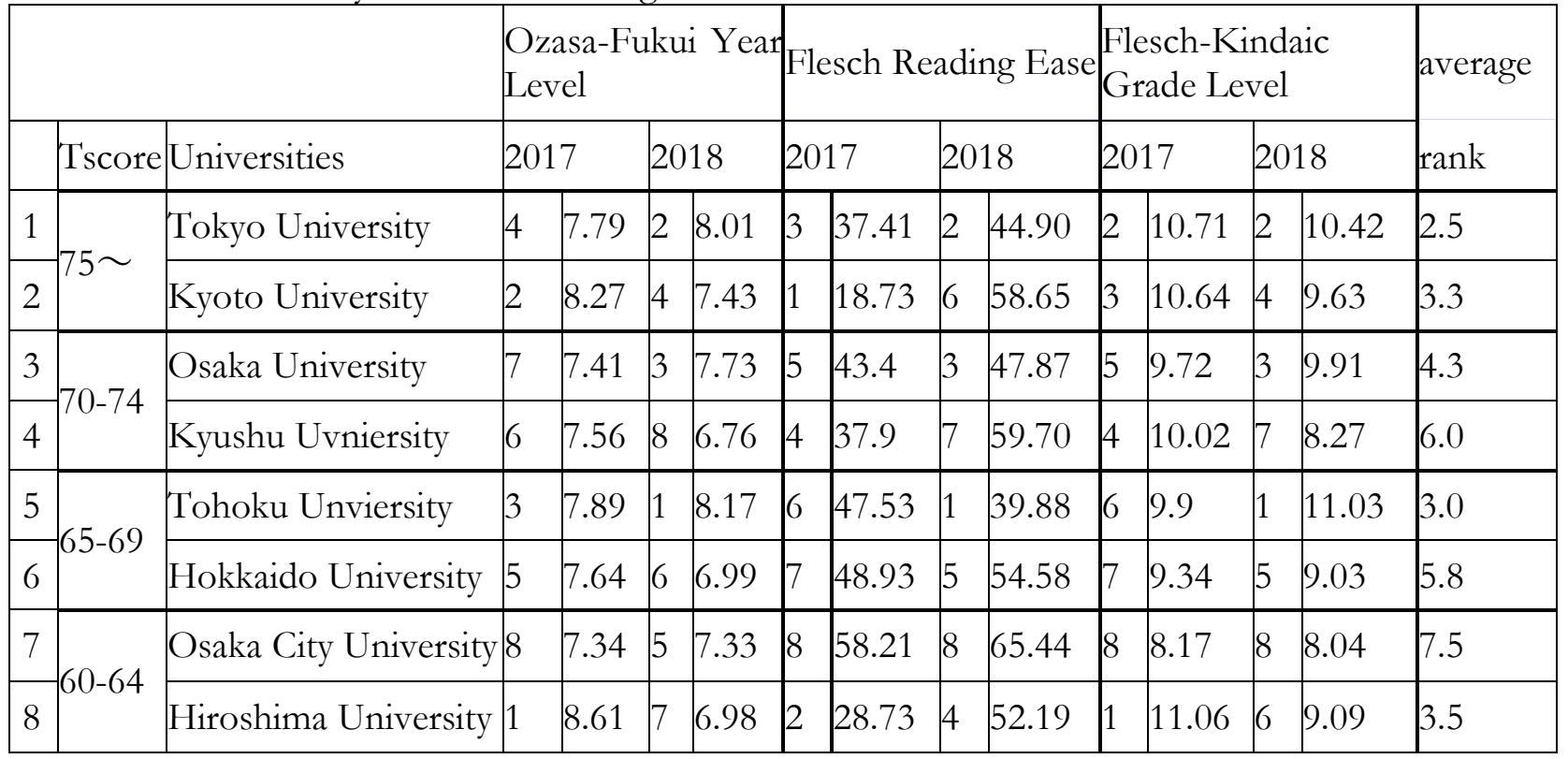


Since the current study added 2018 exams to the 2017 data, we looked at the readability results in the two-year span. Table 3 shows its results. The left columns of readability scores show the rankings according to the scores. It is clear that the rankings of the three measurements do not coincide with the T-score order. It is also clear that the 2017 ranking and 2018 ranking do not match in most of the schools with the exception of Tokyo University and Osaka City University. As "average rank" shows Tokyo University maintains the first place and Osaka City University remains to be the lowest ranked. These results seem to indicate that Tokyo University and Osaka City University may have some strong features in their English exams, which have not changed in at least a two year-time i.e. the past two years.

As for the results of the comparison with JACET 8000, the graphs clearly show the 8 exams in both 2017 and 2018 are basically in the same pattern: most words are placed in Level 1 followed by Level 2, with a bit more words in Level 8 and above. The results indicate that all exams' vocabulary is somehow controlled.

\section{Correspondence Analysis}

\section{Results and Discussion of 16 exams (2017 \& 2018 exams)}

Correspondence analysis summarizes a set of data, in this case, the eight exams and the Center test, and produces eight categories based on the word frequency. The categories are named as "dimensions."

We first create a word frequency table of the eight texts (eight exams) on an excel sheet. We then take out personal and proper nouns and choose the top 100 words to create a cross tabulation (also called "contingency table") in which the columns contain the number of frequency and rows with the names of nine texts. We then compute the data into Correspondence Analysis through a statistical analysis tool (we used College Analysis developed by Dr. M. Fukui). Correspondence Analysis (CA) computes the tabulation, weighs its rows and columns to make an orthogonal computation and produces 15 dimensions.

Figure 1 is a bi-plot map based on Dimension 1 and 2 results; the horizontal bar places Dimension 1 results and the vertical bar places Dimension 2 results. The cumulative contribution rate in Table 4 suggests combined data of Dimension 1 and 2 explains 32.7\% of the entire relationship of 16 exams.

Table 4: Correspondence Analysis Results

\begin{tabular}{|c|c|c|c|c|c|c|c|c|c|c|c|c|c|c|c|}
\hline & Dim 1 & $\begin{array}{l}\operatorname{Dim} \\
2\end{array}$ & $\begin{array}{l}\operatorname{Dim} \\
3\end{array}$ & $\begin{array}{l}\text { Dim } \\
4\end{array}$ & $\begin{array}{l}\operatorname{Dim} \\
5\end{array}$ & $\begin{array}{l}\operatorname{Dim} \\
6\end{array}$ & Dim & $\begin{array}{l}\text { Dim } \\
8\end{array}$ & $\begin{array}{l}\text { Dim } \\
9\end{array}$ & $\begin{array}{l}\text { Dim } \\
10\end{array}$ & $\begin{array}{l}\text { Dim } \\
11\end{array}$ & $\begin{array}{l}\text { Dim } \\
12\end{array}$ & $\begin{array}{l}\operatorname{Dim} \\
13\end{array}$ & $\begin{array}{l}\operatorname{Dim} \\
14\end{array}$ & $\begin{array}{l}\text { Dim } \\
15\end{array}$ \\
\hline Eigen value & 0.149 & 0.135 & 0.092 & 0.079 & 0.067 & 0.06 & 0.054 & 0.045 & 0.043 & 0.035 & 0.031 & 0.025 & 0.023 & 0.017 & 70.012 \\
\hline correlation & 0.386 & 0.367 & 0.303 & 0.281 & 0.258 & 0.246 & 0.233 & 0.212 & 0.206 & 0.187 & 0.175 & 0.159 & 0.151 & 0.129 & 0.111 \\
\hline $\begin{array}{l}\text { contribution } \\
\text { rate }\end{array}$ & 0.172 & 0.156 & 0.106 & 0.091 & 0.077 & 0.07 & 0.063 & 0.052 & 0.049 & 0.041 & 0.035 & 0.029 & 0.026 & 50.019 & 0.014 \\
\hline $\begin{array}{l}\text { cumulative } \\
\text { contribution } \\
\text { rate }\end{array}$ & 0.172 & 0.327 & 0.434 & 0.525 & 0.602 & 0.67 & 0.735 & 0.78 & 0.83 & 0.876 & 0.911 & 0.94 & 0.967 & 0.986 & \\
\hline
\end{tabular}

Dim $=$ dimension

On the bi-plot map of Figure 1 Osaka-City University's 2018 exam is placed solely on the far corner of the horizontal bar suggesting that the exam is very different from other exams. 
Figure 1: Bi-plot map of Dimension $1 \& 2$

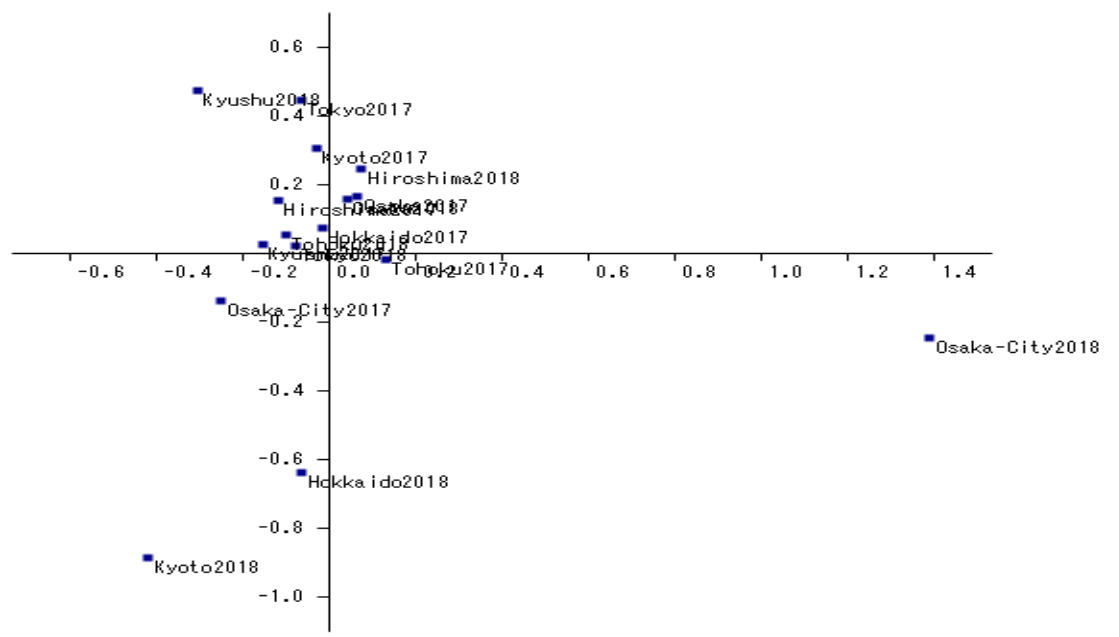

The left column of Dim 1 in Table 5 shows the list of words which have contributed in pushing up Osaka City U 2018 exam to the top. We find personal pronouns such as "me," "I," and "his" among the top words of the list, which suggest that the exam contains many first pronouns in comparison with other exams.

However, the large value of Osaka-City U may have pushed the rest of the schools much closer to each other than a case without Osaka-City U. In other words, the results may not clearly depict the interrelationships among the universities. Having considered this possible flaw, we have decided to try another CA with data without Osaka-City U exams.

Table 5: Top words list of Dimension $1 \& 2$

$0.172 \operatorname{Dim} 1$

\begin{tabular}{|l|l|}
\hline me & 3.299 \\
\hline I & 2.257 \\
\hline his & 1.421 \\
\hline had & 1.396 \\
\hline over & 1.191 \\
\hline at & 0.683 \\
\hline were & 0.573 \\
\hline make & 0.447 \\
\hline only & 0.435 \\
\hline was & 0.431 \\
\hline other & 0.427 \\
\hline
\end{tabular}

0.156

\begin{tabular}{|l|l|}
\hline help & -0.804 \\
\hline you & -0.746 \\
\hline planet & -0.648 \\
\hline she & -0.631 \\
\hline others & -0.624 \\
\hline her & -0.575 \\
\hline while & -0.507 \\
\hline how & -0.484 \\
\hline
\end{tabular}

\begin{tabular}{|l|l|}
\hline planet & 1.192 \\
\hline business & 1.09 \\
\hline desertification & 0.833 \\
\hline than & 0.736 \\
\hline different & 0.608 \\
\hline years & 0.6 \\
\hline no & 0.594 \\
\hline may & 0.592 \\
\hline know & 0.574 \\
\hline
\end{tabular}

Dim 2

\begin{tabular}{|l|l|}
\hline she & -2.009 \\
\hline her & -1.761 \\
\hline help & -1.482 \\
\hline others & -1.294 \\
\hline you & -0.965 \\
\hline could & -0.834 \\
\hline while & -0.728 \\
\hline had & -0.701 \\
\hline me & -0.617 \\
\hline if & -0.611 \\
\hline
\end{tabular}




\section{Results and Discussion of CA without Osaka-City University (14 exams)}

Table 6 shows the results of correspondence analysis without Osaka-City University. The cumulative contribution rate up to Dimension 2 is 0.318 , which is very close to that of CA with Osaka-City University (see Table 4).

Table 6: Results of Correspondence Analysis without Osaka-City U

\begin{tabular}{|l|l|l|l|l|l|l|l|l|l|l|l|l|l|}
\hline $\begin{array}{l}2017-18 \\
\text { without } \\
\text { Osaka-C }\end{array}$ & Dim 1 & $\operatorname{Dim} 2$ & $\operatorname{Dim} 3$ & $\operatorname{Dim} 4$ & $\operatorname{Dim} 5$ & $\operatorname{Dim} 6$ & $\operatorname{Dim} 7$ & $\operatorname{Dim} 8$ & $\operatorname{Dim} 9$ & $\begin{array}{l}\operatorname{Dim} \\
10\end{array}$ & $\begin{array}{l}\operatorname{Dim} \\
11\end{array}$ & $\begin{array}{l}\operatorname{Dim} \\
12\end{array}$ & $\begin{array}{l}\operatorname{Dim} \\
13\end{array}$ \\
\hline eigenvalue & 0.147 & 0.098 & 0.086 & 0.074 & 0.065 & 0.061 & 0.054 & 0.044 & 0.039 & 0.032 & 0.032 & 0.022 & 0.017 \\
\hline correlation & 0.383 & 0.313 & 0.293 & 0.271 & 0.255 & 0.247 & 0.231 & 0.21 & 0.197 & 0.18 & 0.178 & 0.15 & 0.129 \\
\hline $\begin{array}{l}\text { contributio } \\
\text { n rate }\end{array}$ & 0.191 & 0.127 & 0.112 & 0.095 & 0.085 & 0.079 & 0.069 & 0.057 & 0.05 & 0.042 & 0.041 & 0.029 & 0.022 \\
\hline $\begin{array}{l}\text { cumulative } \\
\text { contributio } \\
\text { n rate }\end{array}$ & 0.191 & 0.318 & 0.43 & 0.525 & 0.61 & 0.689 & 0.759 & 0.816 & 0.866 & 0.908 & 0.949 & 0.978 & 1 \\
\hline
\end{tabular}

Figure 2 shows the bi-plot map based on the results of the dimensions $1 \& 2$ of the CA without Osaka-City University. When compared with Figure 1 map, it is obvious that the exams are spread over a wider area of the map rather than being crammed up along both vertical and horizontal bars, which, we believe, shows a more accurate relationship of 14 exams than the previous analysis with 16 exams.

The map shows that Hokkaido 2018 and Tokyo 2017 are placed notably away from each other. Hokkaido on the left side and Tokyo right side of the horizontal bar. Moreover, it shows that Kyoto 2018 is placed away from other exams both vertically and horizontally. Kyushu 2018 is also placed vertically farther down on the vertical bar. It is very clear from the map that Tokyo 2107, Hokkaido 2018, Kyoto 2018 and Kyusyu 2018 have some unique features when compared with other exams.

Figure 2: Bi-plot map of Dimension 1 \& 2 (CA without Osaka-City U)

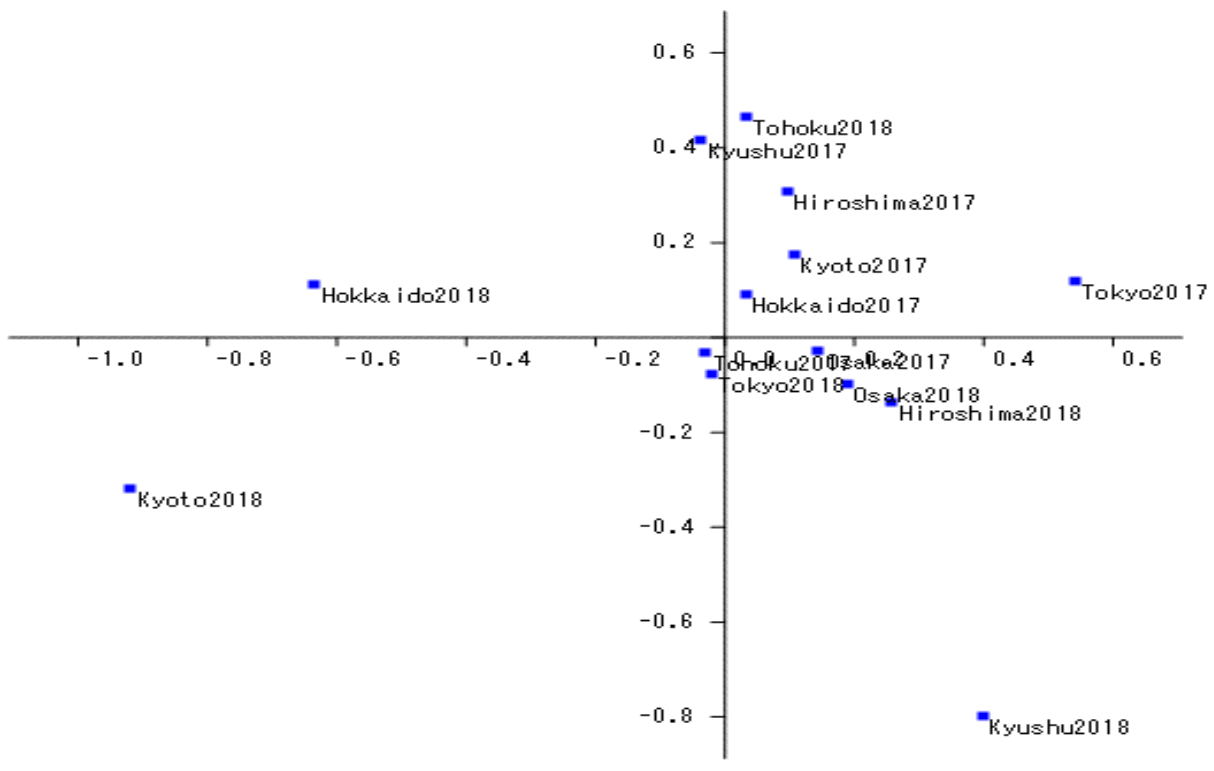


The bi-plot map of Figure 2 is based on the data of dimensions 1 and 2: the list of dimension 1 is mapped on the horizontal axis and dimension 2 list on the vertical axis.

Kyoto 2018 and Hookaido 2018 are placed on the minus side of the horizontal axis (see Fig. 2), and Table 8 shows their exact values: -0.919 for Kyoto 18 and -0.634 for Hokkaido 2018. Kyushu 2017 follows Hokkaido 2018, but its value is mere 0.037. The difference in value between Hokkaido 2018 and Kyushu 2017 is 0.597; a rather big difference.

Tokyo 2017 is placed on the plus side of the horizontal axis away from other exams. But dimension 1's breakdown list in Table 8 shows that the difference between Tokyo 2017 (0.541) and Kyushu 2018 (0.397) is only 0.134. On the vertical axis of Fig. 2, Kyusyu 2018 is placed on the minus side away from other exams. Dimension 2 breakdown shows Kyushu 2018 has -0.798 and has 0.479 difference from Kyoto 2018 (0.319), the second largest value in the list. The difference is notably large. Thus, this breakdown indicates that Tokyo 2017, Kyushu 2018, Kyoto 2018 and Hokkaido 2018 have unique features distinguishing it from other exams. Kyushu 2018 also has some unique features but less distinct.

Table 7 shows the lists of top words that constitute dimensions 1 and 2. On the list of dimension 1, "business" and "culture" have higher values $(1.24 ; 1.222)$ than the rest on the list. Both words are used in Tokyo17 and they are obviously the keywords for placing Tokyo17 at the top of Dimension 1 list (see Table 8 and Table 10). The exam text of Tokyo17 describes culture and business. Based on more than 500 studies over a period of 35 years, it proposes that occupation and socioeconomic status are strong factors of work values than "culture."

On the minus side of Table 9, "she," "her," "help," "you," and "others" have notably high values $(-1.967 \sim-1.332)$, thus these are the keywords to contribute for placing Kyoto18 and Hokkaido18 on the top of the minus side of Dimension 1 (see Table 8). Kyoto18 writes on a theme of "helping others," in which it gives an example of a woman in her eighties. Hokkaido18 describes the life of Grace Hopper, a well-known mathematician and created one of the first computer languages. She was also a high-ranking Navy officer. It is now clear that "woman" is a key theme in both exam texts of Kyoto19 and Hokkaido18. Based on these findings from the list of words of Dimension 1, it is safe to conclude that Dimension 1 categorizes the exams based on socioeconomic viewpoints in which "business and culture" and "gender" are found as polar factors.

Table 7: Top words list

without Osaka-City

\begin{tabular}{|l|l|l|l|l|}
\hline & Dim 1 \\
\hline business & 1.24 \\
\hline culture & 1.222 \\
\hline planet & 0.967 \\
\hline than & 0.818 \\
\hline no & 0.644 \\
\hline different & 0.6 \\
\hline human & 0.555 \\
\hline could & -0.901 \\
\hline much & 0.541 \\
\hline
\end{tabular} \begin{tabular}{|l|l|}
\hline you & -1.343 \\
\hline help & -1.614 \\
\hline her & -1.718 \\
\hline she & -1.967 \\
\hline
\end{tabular}




\begin{tabular}{|l|l|}
\hline animals & 0.527 \\
\hline research & 0.522 \\
\hline may & 0.511 \\
\hline life & 0.51 \\
\hline
\end{tabular}

without Osaka-City

\begin{tabular}{|c|c|c|c|}
\hline & $\operatorname{Dim} 2$ & been & -0.522 \\
\hline students & 1.241 & know & -0.526 \\
\hline family & 1.176 & those & -0.566 \\
\hline will & 0.882 & we & -0.597 \\
\hline work & 0.763 & into & -0.617 \\
\hline common & 0.666 & you & -0.624 \\
\hline \multirow[t]{6}{*}{ their } & 0.513 & our & -0.677 \\
\hline & & so & -0.684 \\
\hline & & how & -0.717 \\
\hline & & there & -0.902 \\
\hline & & life & -1.097 \\
\hline & & planet & -2.227 \\
\hline
\end{tabular}

On Dimension 2 list of Table 7, "students" and "family" are listed on top on the plus side, and "planet," "life," "there" and "how" are some of the top words on the minus side. Table 10 shows schools containing these words. On the plus side, only Kyushu17 has the word "students" hence the high value (15.257), and as for "family," the next high value, Tohoku18 has the highest value. These words with high values explain why Tohoku18 and Kyusyu 17 are placed on top of the list in Dimension 2 of Table 8. Tohoku18 exam text describes the role of an aunt and an uncle in a family, hence the high value of "family." Moreover, Kyusyu17 writes about Problem Based Learning in universities in relation to interdisciplinary studies, hence high use of the word "students."

On the minus side list of Dimension 2 (Table 8), "planet," "life," "there" and "how" are some of the prominent words. In addition, we find that these words appear mostly in Kyushu18 and Kyoto18. Kyusyu18 writes about planet Mars; its history with our culture and the question of life on Mars. "Planet" and "life" are related directly to this theme, and "there" is mostly used to describe the planet in the text.

Describing features of Dimension 2 is not as simple as those of Dimension 1 because of the topics chosen by Tohoku18 and Kyushu17, the first and second schools on the plus side list have seemingly very few things in common; Tohoku18 writes on a theme of the role of aunt and uncle in family and Kyushu17 describes interdisciplinary study programs such as Problem Based Learning. Tohoku17 is a sociological theme and Kyusyu17 is an educational theme. 
Furthermore, their values are not so wide apart as to conclude one is overwhelmingly strong than the other: Tohoku18 has 0.464 and Kyushu17 0.417 (Table 8). However, Hiroshima17, the third on the list, deals with the importance of studying abroad particularly for Americans, which could suggest that overall feature of the plus side of Dimension 2 is "educational" related.

On the other hand, features of the plus side are seemingly less complicated since Kyusyu18, the top on the list outscores the second school, Kyoto18 (-0.798 and -0.319). It is safe to conclude that the feature of Kyusyu18 is a deciding factor for the minus side. Based on these observations, we conclude that the plus side of Dimension 2 features "education," and "astronomical science" for the minus side.

Table 10: Top words in dimension 1

\begin{tabular}{|c|c|c|c|c|c|c|c|c|c|c|c|c|c|c|c|}
\hline & Dim 1 & Tokyo18 & \begin{tabular}{|l|} 
Tokyo17 \\
\end{tabular} & Kyoto18 & Kyoto17 & Osaka18 & $\begin{array}{l}\text { Osaka17 } \\
\end{array}$ & Kyushu18 & \begin{tabular}{|l|} 
Kyushu17 \\
\end{tabular} & Tohoku 18 & Tohoku17 & Hokkaido 18 & \begin{tabular}{|l|} 
Hokkaido 17 \\
\end{tabular} & Hiroshima 18 & Hiroshima 17 \\
\hline business & 1.24 & 0 & 17.647 & 0 & 0 & 0 & 1.433 & 0 & 0 & 0 & 0 & 0 & 1.558 & 0 & \\
\hline culture & 1.222 & 0 & 14.706 & 0 & 0 & 0 & 1.433 & 1.842 & 0 & 1.099 & 0 & 0 & 0 & 0 & 0 \\
\hline planet & 0.967 & 0 & 0 & 0 & 0 & 0 & 1.433 & 20.258 & 0 & 0 & 0 & 0 & 0 & 1.942 & 0 \\
\hline than & 0.818 & 0.864 & 17.647 & 0 & 1.969 & 2.778 & 2.865 & 7.366 & 0 & 1.099 & 2.041 & 0 & 1.558 & 1.942 & 7.156 \\
\hline no & 0.644 & 0 & 5.882 & 0 & 1.969 & 2.778 & 1.433 & 0 & 1.387 & 1.099 & 2.041 & 0 & 0 & 3.883 & 0 \\
\hline \begin{tabular}{|l} 
different \\
\end{tabular} & 0.6 & 4.318 & 8.824 & 0 & 1.969 & 5.556 & 4.298 & 0 & 0 & 0 & 1.02 & 0 & 3.115 & 1.942 & \\
\hline human & 0.555 & 0 & 2.941 & 0 & 0 & 4.167 & 0 & 0 & 0 & 0 & 0 & 0 & 6.231 & 5.825 & 0 \\
\hline much & 0.541 & 0 & 8.824 & $\begin{array}{l}1.818 \\
\end{array}$ & 3.937 & 0 & 0 & 5.525 & 1.387 & 0 & 0 & 1.445 & 0 & 3.883 & 1.789 \\
\hline animals & 0.527 & 0 & 0 & 0 & 0 & 16.667 & 0 & 0 & 0 & 0 & 0 & 0 & 0 & 3.883 & 0 \\
\hline research & 0.522 & 1.727 & 2.941 & 0 & 1.969 & 4.167 & 0 & 3.683 & 2.774 & 0 & 2.041 & 0 & 0 & 0 & 0 \\
\hline may & 0.511 & 6.045 & 2.941 & 0 & 0 & 2.778 & 0 & 11.05 & 1.387 & 5.495 & 0 & 0 & 3.115 & 0 & 1.789 \\
\hline life & 0.51 & 0.864 & 2.941 & \begin{tabular}{|l|}
1.818 \\
\end{tabular} & 0 & 0 & 1.433 & 9.208 & 0 & 2.198 & 1.02 & 0 & 0 & 0 & 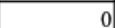 \\
\hline
\end{tabular}

\begin{tabular}{|c|c|c|c|c|c|c|c|c|c|c|c|c|c|c|c|}
\hline & $\operatorname{Dim} 1$ & Tokyo18 & Tokyol7 & Kyoto 18 & Kyoto17 & Osaka18 & Osaka17 & Kyushul8 & Kyushul7 & Tohoku18 & Tohoku17 & Hokkaido18 & Hokkaidol7 & Hiroshima18 & Hiroshima17 \\
\hline one & -0.501 & 2.591 & 2.941 & 3.636 & 1.969 & 2.778 & 2865 & 1.842 & 2774 & 5.495 & 2.041 & 15.896 & 1.558 & 0 & 3.578 \\
\hline had & -0.778 & 2.591 & 0 & 3.636 & 3.937 & 1.389 & 0 & 0 & 0 & 0 & 4.082 & 8.671 & 0 & 1.942 & 0 \\
\hline could & -0.9 & 0.864 & 0 & 7.273 & 0 & 1.389 & 7.163 & 0 & 0 & 0 & 1.02 & 4.335 & 1.558 & 0 & 0 \\
\hline others & -1.332 & 4.318 & 0 & 12727 & 0 & 0 & 0 & 0 & 0 & 0 & 1.02 & 1.445 & 3.115 & 0 & 1.789 \\
\hline you & -1.343 & 0.864 & 0 & 20 & 0 & 0 & 0 & \begin{tabular}{|l|l|}
3.683 \\
\end{tabular} & 6.935 & 0 & 2.041 & 0 & 0 & 0 & 0 \\
\hline help & -1.614 & 0.864 & 0 & 20 & 0 & 0 & 0 & 0 & 2.774 & 1.099 & 1.02 & 0 & 0 & 0 & 3.578 \\
\hline her & -1.718 & 0 & 2.941 & 30.909 & 0 & 0 & 0 & 0 & 0 & 1.099 & 5.102 & 23.121 & 0 & 0 & 0 \\
\hline she & -1.967 & 0 & 0 & 25.455 & 0 & 0 & 0 & 0 & 0 & 1.099 & 1.02 & 21.676 & 0 & 0 & 0 \\
\hline
\end{tabular}

\section{Cluster Analysis}

The cumulative contribution rate of Dimension 1 and 2 is 0.319 (see Table 6), meaning Dimension 1 and 2 would explain a mere $31.9 \%$ of the total analysis result. It is obviously not sufficient to describe the total relationship among 14 exams. We, therefore, conducted Cluster analysis since this analysis displays all 13 dimensions on a dendrogram.

\section{Results}

Figure 3 is a dendrogram based on the results produced by Cluster analysis. It graphically depicts the distance relationship of the 14 exams based on the 13 dimensions produced by Correspondence analysis (see Table 6). 
Figure 3: Dendrogram without Osaka-City U

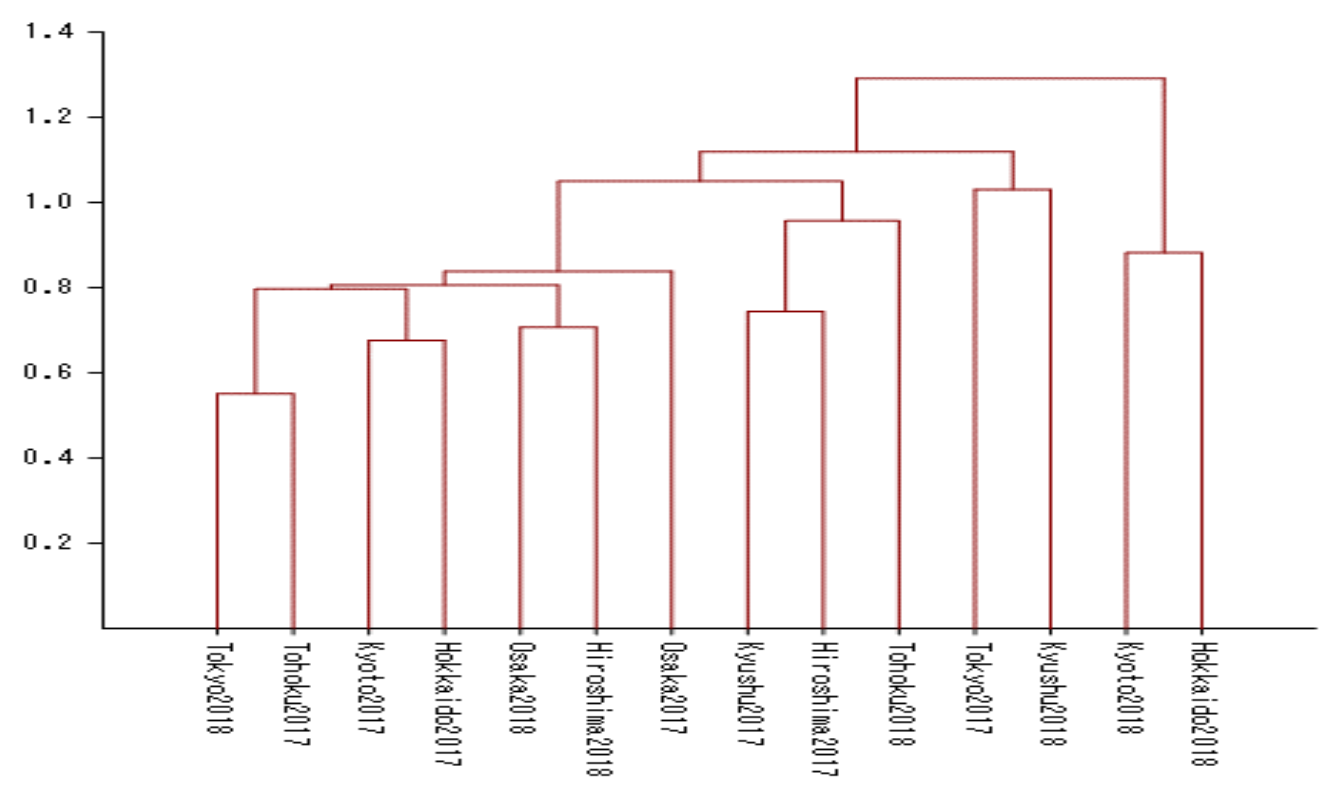

On the dendrogram, Hokkaido18 and Kyoto18 are paired and placed on the farthest right, suggesting that these two exams have some common features unique among the 14 exams. Kyusyu18 and Tokyo17 are also paired and placed next to the Hokkaido and Kyoto pair.

\section{Discussion}

Hokkaido18 and Kyoto18 both write on "woman": Hokkaido18 describes a life of Grace Hopper, a high-rank Navy officer and one of the first to create computer languages. In addition, Kyoto18 writes about a psychological topic of "helping others" in which it gives a story an eighty-year-old woman. Kyusyu18 writes about the planet Mars, cultural interest as well as scientific interest towards the planet. And Tokyo17 writes on culture and country: occupation and socioeconomic status are better predictors of work values than the country of origin.

CA also finds these four exams side by side in the list of Dimension 1. Dimension 1 list places Tokyo17 and Kyusyu 18 on the top of the plus side. Kyoto18 and Hokkaido18 are placed on the top of the minus side. As we pointed out in the discussion of CA, Kyoto18 (-0.919) and Hokkaido18 (-0.634) have much higher values than the third place Kyusyu17 (-0.037). Tokyo17 (0.541) and Kyusyu18 (0.397) also have higher values than the third place Hiroshima18 (0.258), but not as obvious as Kyoto18 and Hokkaido18, hence second pair from the right on the dendrogram.

The results of Cluster analysis show the fact that the bi-plot map of Correspondence analysis, which are based on Dimension 1 and 2, shows some similar results, in that Kyoto18 and Hokkaido18 exams have strong features which stand out among 14 exams. The value of Kyoto18 in Dimension $1(-0.919)$ is the highest value in Dimension 1 and 2. What both Correspondence analysis and Cluster analysis results, however, have revealed is that when the national universities' exams for two years are statistically compared, other than topics of the exams, the exams could not be categorized.

\section{Conclusion}

We have selected English exam data from eight universities, the same universities as Watanabe \& Fukui (2018) as a follow up study. In particular, we have set the following three research questions: 
RQ1: What are the 2018 exams' readability levels?

RQ2: Are there any common characteristics between 2017 and 2018 exams of the same schools? RQ3: Are there any characteristics unique to 2017 or to 2018 ?

As for the first RQ, OFYL results showed that all exams levels are higher than high school third year (Grade 12). This result was not surprising because 2017 exams of the same schools produced very similar results (Watanabe \& Fukui, 2018). Flesch Reading Ease and FleschKincaid Grade Level, readability measurement tools used in America also showed the exams are at high levels even in an American standard. The result once again pointed out a fact that the entrance exams of national universities would require English competency much higher than the level of high school English textbooks. On the other hand, when compared with JACET8000, their vocabulary levels follow very similar patterns, which would indicate their vocabulary is controlled.

As for RQ2, we employed Correspondence Analysis to study the characteristics of 8 exams in terms of the interrelationship among them. However, the results showed that because of the high value of Osaka-City University, the rest of the schools' exams are placed around the center of the bi-plot map, not showing the interrelationships among the rest of the universities. We, therefore, conducted another analysis without Osaka-City University. The results of the correspondence analysis without Osaka-City U revealed that Kyoto18 and Hokkaido18 feature gender, depicting women's accounts, and Tokyo17 and Kyusyu18 feature business and culture. And they revealed that Kyusyu18's topic, describing Planet Mars, is very unique among the seven universities.

As for RQ3, we found that contrary to our expectations there are no strong ties between 2017 and 2018 exams even within the same school. In other words, there are no specific features or characteristics that are succeeded to another year within a school, at least from the statistical points. The cluster analysis based on the seven dimensions' values showed that Kyoto18 and Hokkaido18, both of which depict women's life, have strong ties to each other. We conclude that the study has revealed some unique features of entrance exams. However, we must admit that the features found in the study are not correlated with school or year. In other words, we cannot find any features unique to each school or year. We intend to make further studies on the entrance exams for next year to see if we have different results.

\section{References}

Benesse. (2018). The list of national universities according to T-score. Retrieved from http://kou.benesse.co.jp/hensachi/search.php.

Fukui, M., \& Ozasa, T. (2017). Ozasa-Fukui Year Level, a Readability Measuring Tool: Its System and Measuring Program. Journal of ICATLL Japan, 4, 1-12.

Ide, H. (2012). Higashi Asia no Daigaku Nyushi Mondai Bunseki, Kawaijuku Guideline. Retrieved from http://www.keinet.ne.jp/gl/12/04/toku2_1204.pdf.

Ishikawa, S., Maeda, T., \& Yamazaki, M. (2010). Gengo Kenkyu no tame no Toke Nyumon, Kuroshio Shuppan: Tokyo.

Ministry of Education. (2010). Ministry of Education. Retrieved from http://www.mext.go.jp/b_menu/toukei/002/002b/1368900.htm.

Ozasa, T., \& Abe, N. (2015). Readability of English Textbooks at Primary School in Thailand and Japan. Journal of ICT ATLL Japan, 2, 11-20.

Ozasa T., \& Watanabe, K. (2016). Readability of English Textbooks at Primary and Junior High Schools in Thailand: A Comparison between Thai and Japanese Current Textbooks. Journal of ICTATLL Japan, 3, 1-13.

Ozasa, T., Watanabe, K., Uenishi, K., \& Sakamoto, M. (2017). Readability of EFL Textbooks at Primary School in China: A Comparison with Japanese Current Textbooks. Journal of ICT ATLL Japan, 4, $59-70$. 
Sakamoto, M., Watanabe, K., \& Ozasa, T. (2017). A Correspondence Analysis of Five Japanese Historical Englishas-a-Foreign-Language Textbooks. International Conference on Education, Psychology and Society, Conference Proceedings. July 25-27, Okiwana, Japan, 132-144.

Japan Association of College English Teachers Basic Words Revision Committee. (2016). The New JACET List of 8000 Basic Words. Kirihara-Shoten, Tokyo.

Uenishi, K., Watanabe, K., \& Ozasa, T. (2017). Correspondence Analysis of Three Japanese EFL Textbooks: In Reference to the Improvement of a Criterion Measure for a Readability Tool. International Conference on Education, Psychology, and Learning, Conference Proceedings. August 23-25, 49-60.

Urabe, S. (2010). Eiken Bulletin. Retrieved from https://www.eiken.or.jp/center_for_research/pdf/bulletin/vol22/vol_22p232-p238.pdf.

Watanabe, K., \& Fukui, M. (2018). Quantitative Analysis of Initial Stage English Textbooks in Asia in comparison with textbooks in Japan. 2018 International Symposium on Teaching, Education, and Learning Winter Session, Conference Proceedings. January 23-25, 117-130.

Watanabe, K., \& Fukui, M. (2018). Statistical Characteristics of English Exams of Eight National Universities in Japan. 2018 International Conference on Education and Learning, Conference Proceedings, August 22-24, 2018, Waseda University, 373-386. 